\title{
Increased Circulating Apoptotic CD31+/CD42b-and Activated CD62E+ Endothelial Micro Particles in Coronary Artery Disease
}

\author{
Shuai-Shuai Hu, Hong-Gang Zhang*, Qiu-Ju Zhang and Rui-Juan Xiu \\ Institute of Microcirculation, Peking Union Medical College \& Chinese Academy of Medical Sciences, Key Lab of Microcirculation, National Health and Family Planning \\ Commission, China
}

\begin{abstract}
Objective: To examine the phenotypes of endothelial microparticles and platelet microparticles in order to identify a sensitive endothelial microparticle biomarker for monitoring endothelial dysfunction in patients with coronary artery disease, and to examine the mechanisms underlying endothelial dysfunction.

Methods: Thirty-three coronary artery disease patients and 53 healthy subjects were recruited. The absolute numbers of endothelial microparticles and platelet microparticles were measured by flow cytometry, and the clinical characteristics of patients and healthy subjects were recorded. Spearman's correlation analysis and multiple linear regression analysis were performed to analyse the correlation between endothelial microparticles and platelet microparticles, and between microparticles and clinical data.
\end{abstract}

Results: There was a significant difference in the number of CD31+/CD42b- endothelial microparticles and CD62E endotheial microparticles between patients with coronary artery diease and healthy subjects $(p<0.05)$.

Conclusion: Both endothelial apoptosis and activation play an important role in coronary artery disease, although endothelial apoptosis plays the major role. Endothelial microparticles may be used to monitor endothelial dyfunction in patients with coronary artery disease.

Keywords: Endothelial dysfunction; Endothelial microparticles; Coronary artery disease

\section{Introduction}

Endothelial Dysfunction (ED) is an important pathophysiological mechanism that underlies the development of Coronary Artery Disease (CAD) [1]. ED is also predictive of prognosis and cardiovascular risk [2]. Therefore, it is important to identify new biomarkers that can be used to monitor ED in CAD and to identify the underlying mechanisms. ED is determined by the balance between endothelial activation and endothelial apoptosis. Endothelial Microparticles (EMP) are derived from endothelial cells in response to endothelial activation and apoptosis. Some studies report that CD $31^{+} \mathrm{EMP}$ are shed from apoptotic endothelial cells, whereas $\mathrm{CD}^{2} \mathrm{E}^{+} \mathrm{EMP}$ are shed by activated endothelial cells [3]. In addition, a previous study reported that the levels of EMP are higher in CAD patients than in healthy controls [4]. Therefore, the aims of the present study were to examine the phenotypes of EMP and Platelet Microparticles (PMP) in order to identify a sensitive endothelial microparticle biomarker for monitoring $\mathrm{ED}$ in $\mathrm{CAD}$, and to examine the mechanisms underlying ED.

\section{Materials and Methods}

\section{Clinical study population}

Thirty-three CAD patients and 53 healthy subjects were enrolled. CAD was diagnosed when stenosis ( $\geq 50 \%$ of the lumen diameter) was detected in a major coronary artery (the left main coronary artery, the left anterior descending artery, the left circumflex artery, or the right coronary artery) by coronary arteriography. Patients with a history of inflammatory, chronic renal failure, hepatic or hematologic disorders, or autoimmune or malignant diseases were excluded. Healthy subjects were included if they had no known history of medical illness and a normal physical examination. The study protocol was approved by the Institutional Review Boards and Ethics Committees of the Microcirculation Peking Union Medical College and Chinese Academy of Medical Science. All patients and controls provided verbal informed consent before entering the study.

\section{Isolation and measurement of microparticles}

Blood was drawn into blue-top vacuum tubes containing sodium citrate. Within 1 hour, whole blood ( $3 \mathrm{ml}$ per sample) was centrifuged at $1500 \times \mathrm{g}$ for $10 \mathrm{~min}$ to prepare platelet-rich plasma and then again for $10 \mathrm{~min}$ at $13,000 \times \mathrm{g}$ to obtain platelet-poor plasma. The plasma products were stored at $-20^{\circ} \mathrm{C}$ for 1 week and then at $-80^{\circ} \mathrm{C}$ until analysis. A single thaw cycle did not affect microparticle levels in the plasma.

For the EMP assay, platelet-poor plasma $(50 \mu \mathrm{l})$ was incubated with the following monoclonal antibodies ( $4 \mu$ of each): phycoerythrin (PE)-labeled anti-CD31 (560983; BD Biosciences), PE-anti-CD62E (551145; BD Biosciences), PE-anti-CD144 (VE-Cadherin, 12-1449; eBioscience), and fluorescein isothiocyanate-(FITC)-labeled antiCD51/CD61 (integrin alpha v beta3, 110519; eBioscience). For the PMP assay, platelet-poor plasma $(50 \mu \mathrm{l})$ was incubated with $4 \mu \mathrm{l}$ of FTIC-anti-CD42b (555472; BD Biosciences). All samples were incubated with antibodies at room temperature for $20 \mathrm{~min}$ and then diluted with $1 \mathrm{ml}$ of phosphate-buffered saline buffer prior to analysis in a flow cytometer (Accuri C6; Accuri Cytometers). To ensure analysis of $\mathrm{CD} 31^{+} \mathrm{EMP}$ rather than platelet-derived $\mathrm{CD} 31^{+}$microparticles,

*Corresponding author: Hong-Gang Zhang, Peking Union Medical College and Chinese Academy of Medical Sciences, Beijing, China, Tel: 86+13161126646; E-mail: zhanghg1966126@imc.pumc.edu.cn

Received July 07, 2014; Accepted July 28, 2014; Published August 03, 2014

Citation: Hu SS, Zhang HG, Zhang QJ, Xiu RJ (2014) Increased Circulating Apoptotic CD31+/CD42b- and Activated CD62E + Endothelial Micro Particles in Coronary Artery Disease. J Hypertens 3: 167. doi:10.4172/2167-1095.1000167

Copyright: ( $2014 \mathrm{Hu}$ SS, et al. This is an open-access article distributed under the terms of the Creative Commons Attribution License, which permits unrestricted use, distribution, and reproduction in any medium, provided the original author and source are credited. 
Citation: Hu SS, Zhang HG, Zhang QJ, Xiu RJ (2014) Increased Circulating Apoptotic CD31+/CD42b- and Activated CD62E+ Endothelial Micro Particles in Coronary Artery Disease. J Hypertens 3: 167. doi:10.4172/2167-1095.1000167

$\mathrm{CD} 42 \mathrm{~b}^{-}$microparticles were analysed in platelet-poor plasma. Events calibrated by internal standard beads $(0.8 \mu \mathrm{m}$; Sigma) were identified in forward-scatter and side-scatter modes gated on microparticles, and plotted on one- or two-color fluorescence histograms. The Accuri C6 flow cytometer can calculate the absolute number of microparticles in a sample. An isotype control antibody was used as a negative control in all experiments. EMP were defined as $\mathrm{CD} 31^{+} / \mathrm{CD} 42$, z. PMP were defined as $\mathrm{CD} 31^{+}$or $\mathrm{CD} 42^{+}$. Values are expressed as counts/ $\mu$ l of peripheral blood. The laboratory personnel performing the assays were blinded to all clinical data.

\section{Statistical Analysis}

Normally distributed data are expressed as the mean \pm Standard Deviation (SD) and non-normally distributed data as the median and Inter-Quartile Range (IQR) or number. Variables were evaluated

\begin{tabular}{|l|c|c|c|}
\hline Characteristics & Healthy controls & CAD patients & P value \\
\hline Age $(\mathrm{y})$ & $34.26 \pm 7.09(53)$ & $68.21 \pm 12.786(33)$ & $<0.001$ \\
\hline Gender $($ male/female $)$ & $22 / 31$ & $18 / 15$ & 0.239 \\
\hline $\mathrm{BMI}\left(\mathrm{kg} / \mathrm{m}^{2}\right)$ & $22.45 \pm 1.58(53)$ & $26.29 \pm 3.66(27)$ & $<0.001$ \\
\hline $\mathrm{TC}(\mathrm{mmol} / \mathrm{l})$ & $3.80 \pm 0.39(53)$ & $4.05 \pm 0.75(25)$ & 0.127 \\
\hline $\mathrm{HDL}(\mathrm{mmol} / \mathrm{l})$ & $1.37 \pm 0.68(53)$ & $1.14 \pm 0.28(26)$ & $<0.001$ \\
\hline $\mathrm{LDL}(\mathrm{mmol} / \mathrm{l})$ & $1.94 \pm 0.35(53)$ & $2.37 \pm 0.77(26)$ & 0.011 \\
\hline LnTG $(\mathrm{mmol} / \mathrm{l})$ & $0.39 \pm 0.29(53)$ & $0.46 \pm 0.64(26)$ & 0.599 \\
\hline Medications & & & \\
\hline Platelet inhibitors & 0 & $14 / 33$ & \\
\hline Beta-blockers & 0 & $9 / 33$ & \\
\hline ACE-I/ARB & 0 & $7 / 33$ & \\
\hline CCBs & 0 & $12 / 33$ & \\
\hline
\end{tabular}

Values are expressed as the mean $\pm \mathrm{SD}$ and (number)

CAD: Coronary Artery Disease; BMI: Body Mass Index; DM: Diabetes Mellitus; TC Total Cholesterol; HDL: High Density Lipoprotein; LDL: Low Density Lipoprotein; TG: Triglycerides; CCBs: Calcium Channel Blockers. A P value of $<0.05$ was considered significant

Table 1: Demographic and clinical characteristics of the patients and healthy subjects.

$\mathbf{a}$

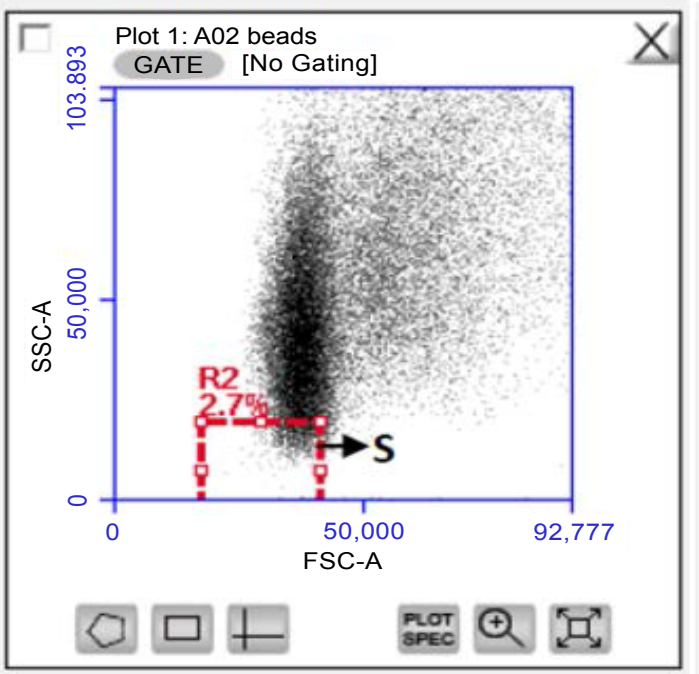

for normality using the Kol-mogorov-Smirnov test. The parametric Student's t-test was used to compare quantitative variables between two groups of normally distributed data, and a non-parametric t-test was used for non-normally distributed data. The Chi-square test was used to compare quantitative and categorical variables. Spearman's correlation analysis and multiple linear regression analysis were used to assess the relationship between different kinds of microparticles. Multiple linear regression analysis was used to determine the relationship between microparticles and clinical and laboratory variables. A P value of $<0.05$ was considered statistically significant. All statistical analyses were performed using SPSS 17.0 software.

\section{Results}

\section{Baseline data}

Baseline data for the 33 patients and 53 healthy subjects are shown in Table 1. Clinical data included age, gender, Body Mass Index (BMI), Total Cholesterol (TC), High Density Lipoprotein (HDL) level, Low Density Lipoprotein (LDL) Level, Triglycerides (TG), and Diabetes Mellitus (DM). Any medications were also noted. Representative flow cytometry plots showing calibration with microparticle beads are shown in Figure 1. The data in Table 1 show that the control subjects were younger than CAD patients $(p<0.001)$ and had a lower BMI $(p<0.001)$. Healthy subjects had higher levels of HDL than the CAD group $(\mathrm{P}<0.001)$, whereas the CAD group had higher levels of LDL $(\mathrm{p}<0.05)$.

\section{Absolue numbers of EMP and PMP in CAD patients and healthy controls}

The number of microparticles in samples from CAD patients and controls is shown in Table 2 and Figure 2. Figure 2 shows that the number of $\mathrm{CD}_{31} 1^{+} \mathrm{CD} 42 \mathrm{~b}^{-} \mathrm{EMP}$ and $\mathrm{CD} 62 \mathrm{E}^{+} \mathrm{EMP}$ was higher in $\mathrm{CAD}$ patients than in healthy controls $(\mathrm{p}<0.001$ and $\mathrm{p}<0.05$, respectively). As shown in Table 2, there was no significant difference in the number of $\mathrm{CD} 144^{+}$and $\mathrm{CD} 51^{+} \mathrm{EMP}$, or the number of CD42 $\mathrm{b}^{+} \mathrm{PMP}$, between CAD patients and controls.

\section{b}

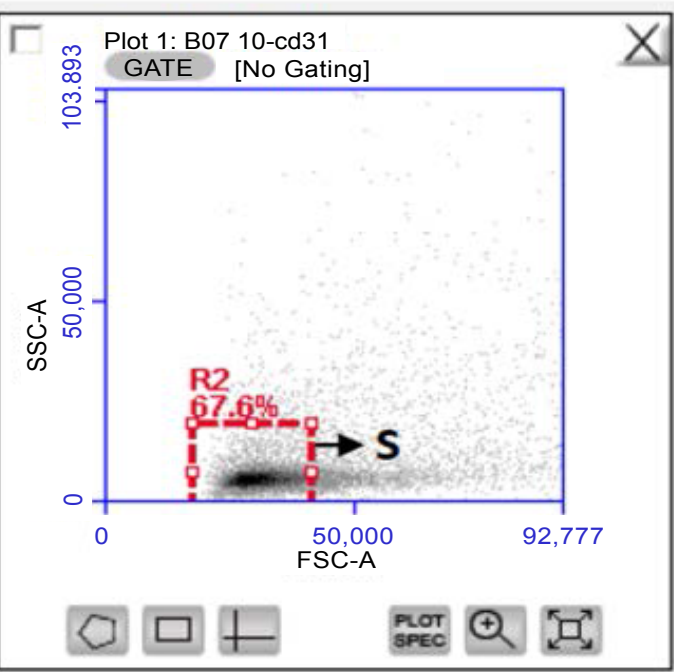

Figure 1: Representive flow cytometry plots of the number of circulating EMP. (a) The $0.8 \mu \mathrm{m}$ calibrator beads, used to define the size of the microparticles, are represented on a side-scatter/forward-scatter dot plot. (b) Circulating microparticles are represented on a side-scatter/forward-scatter dot plot. S window was a gate of MP. 
Citation: Hu SS, Zhang HG, Zhang QJ, Xiu RJ (2014) Increased Circulating Apoptotic CD31+/CD42b- and Activated CD62E+ Endothelial Micro Particles in Coronary Artery Disease. J Hypertens 3: 167. doi:10.4172/2167-1095.1000167

\begin{tabular}{|l|c|c|c|}
\hline Characteristics & Healthy controls & CAD & P value \\
\hline CD31+/CD42b- EMP & $3.81(1.48-9.84)$ & $42.21(8.80-103.63)$ & $<0.001$ \\
\hline CD144+ EMP & $21.13(9.33-36.71)$ & $47.91 \pm 45.74$ & 0.086 \\
\hline CD62E ${ }^{+}$EMP & $2.84 \pm 1.51$ & $4.58 \pm 3.49$ & 0.011 \\
\hline CD51+ EMP & $24.08(8.22-55.79)$ & $26.80 \pm 25.10$ & 0.342 \\
\hline CD42b+ PMP & $38.83(17.14-65.49)$ & $20.26(7.17-54.43)$ & 0.120 \\
\hline
\end{tabular}

Values are expressed as the mean $\pm S D$ or as the median and inter-quartile range CAD: Coronary Artery Disease; EMP: Endothelial Microparticle; PMP: Platelet Microparticle.

A $P$ value $<0.05$ was considered significant

Table 2: Number of microparticles in patients and healthy subjects
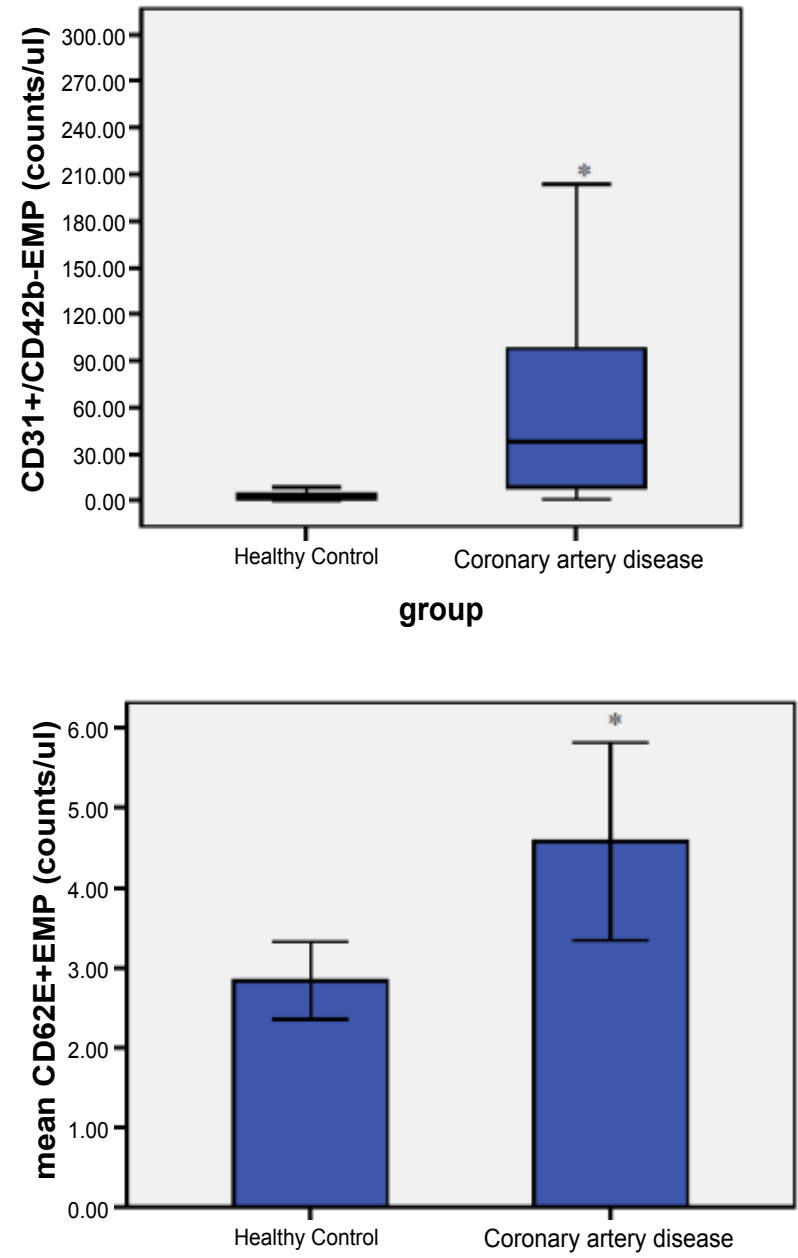

group

Figure 2: Comparison of the number of CD31+ EMP and CD62E EMP in coronary artery disease patients and healthy controls. ${ }^{*} \mathrm{P}<0.05$.

\section{Correlation between different kinds of microparticle}

Figure 3 shows the correlation between different kinds of microparticles, as determined by Spearman's correlation and linear regression analysis. The data revealed a significant correlation between the number of $\mathrm{CD} 31^{+} / \mathrm{CD} 42 \mathrm{~b}-\mathrm{EMP}$ and the number of $\mathrm{CD} 62 \mathrm{E}^{+} \mathrm{EMP}$ $(\mathrm{p}=0.044 ; \mathrm{r}=0.524)$.

\section{Correlation between microparticles and clinical parameters}

Multiple linear regression analysis was used to evaluate the relationship between independent variables (age, gender, BMI, TC, DM, HDL, LDL and TG) and dependent variables $\left(\ln \left(\mathrm{CD} 31^{+} \mathrm{EMP}\right)\right.$ and $\ln (\mathrm{CD} 62 \mathrm{E}$ EMP)). Because microparticle values were not normally distributed, we examined $\ln$ (microparticle) values. The results showed that neither $\mathrm{CD}_{2} \mathrm{E}^{+} \mathrm{EMP}$ nor $\mathrm{CD} 31^{+} \mathrm{EMP}$ correlated with clinical parameters.

\section{Discussion}

The major finding of this study is that endothelial activation and apoptosis, reflected by increased numbers of microparticles released from activated $\left(\mathrm{CD}^{2} \mathrm{E}^{+}\right)$and apoptotic $\left(\mathrm{CD} 31^{+}\right)$endothelial cells, play an important role in CAD. These data provide insights into the mechanisms underlying endothelial dysfunction in CAD. There were no significant differences between the numbers of other types of EMP or PMP between patients and healthy subjects.

ED is a critical factor in the development and progression of CAD. EMP are membrane vesicles that are shed from endothelial cells in response to injury, activation, or apoptosis. EMP retain endothelial cell surface proteins and components of the cytosol, including enzymes, RNA, and micro (mi)RNA. The biological functions of EMP are related to inflammation, vascular tone, and coagulation $[5,6]$. The results of the present study show that the number of EMP is higher in CAD patients than in healthy controls, which is an indicator of endothelial apoptosis and activation in these patients. These results complement and extend the important findings of Nioks Werner et al. [7], who demonstrated that apoptotic EMP are involved in the pathogenesis of $\mathrm{ED}$ in CAD. A previous study showed that a $\mathrm{CD} 62 \mathrm{E}^{+} / \mathrm{CD} 31^{+}$ratio $\leq 1.0$ suggests that endothelial cell apoptosis plays the main role in the vessel damage associated with CAD [8]. Here, we showed that the $\mathrm{CD}^{2} \mathrm{E}^{+} /$ $\mathrm{CD} 31^{+}$ratio in the $\mathrm{CAD}$ group was $<1.0$; significantly lower than that in the healthy group $(\mathrm{p}<0.001)$. Therefore, these results confirm that endothelial apoptosis plays the major role in the development of CAD.

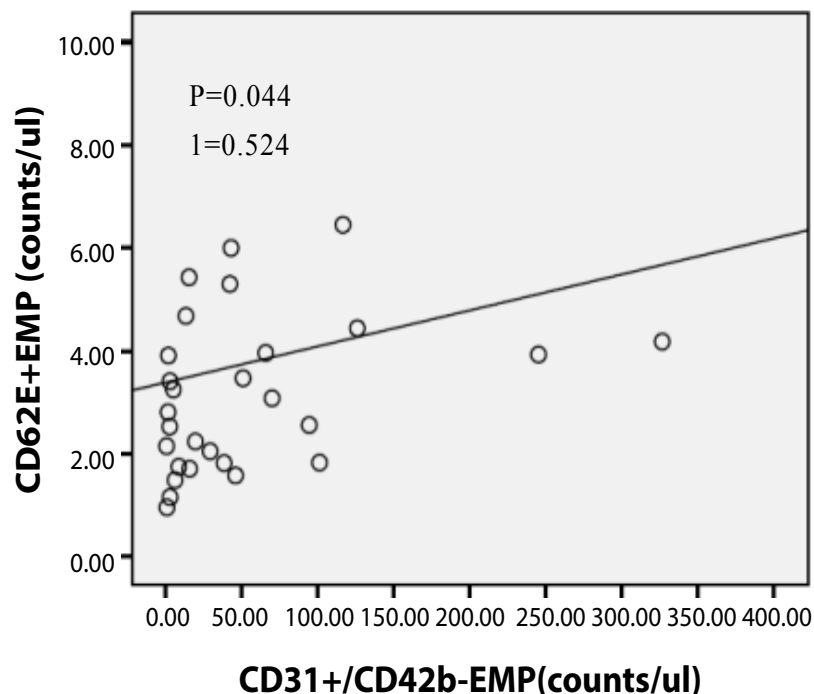

Figure 3: Correlation between different types of microparticle. Statistical significance was determined by linear regression analysis. $R$ values were determined by Spearman's correlation analysis. 
Citation: Hu SS, Zhang HG, Zhang QJ, Xiu RJ (2014) Increased Circulating Apoptotic CD31+/CD42b- and Activated CD62E+ Endothelial Micro Particles in Coronary Artery Disease. J Hypertens 3: 167. doi:10.4172/2167-1095.1000167

Page 4 of 4

Previous studies report that CAD patients have higher numbers of PMP $[9,10]$ than healthy subjects; however, our data did not support this. The reasons for this may be 2-fold: (1) Leon Bernal-Mizrachi and his team [11] found that the number of PMP in patients with stable angina was no different from that in healthy subjects, but there was a difference between subjects who had experienced myocardial infarction and those with unstable angina. Thus, it appears that the number of PMP is related to the type of CAD (further experiments are needed to examine the role of PMP in different types of CAD); (2) Kiat et al. [12] reported that an increase in the number of PMP was not related to the extent of CAD. Therefore, the role of PMP in CAD requires further study; in the meantime, EMP may be more suitable as a biomarker for ED. Although the results in this paper are promising, two study limitations should be taken into account. One is that the sample sizes were small. The second is that the experimental approach was quite simplistic. Further experiments must be designed to examine the detailed mechanisms underlying the role of microparticles in CAD.

In conclusion, the results of the present study suggest that endothelial cell apoptosis plays the major role in the development of CAD. Therefore, it seems reasonable to suggest that EMPs have potential as biomarkers for monitoring ED. Future studies should aim to develop agents that inhibit EMP formation.

\section{Acknowledgement}

This study was supported by the National Natural Science Foundation of China (grant no. 11274046) and the Peking Union Medical College Innovation Funding (no. 3332013021)

\section{References}

1. Manganaro A, Ciracì L, Andrè L, Trio O, Manganaro R, et al. (2014) Endothelial Dysfunction in Patients With Coronary Artery Disease: Insights From a FlowMediated Dilation Study. Clin Appl Thromb Hemost .
2. Hafner F, Kieninger A, Meinitzer A, Gary T, Froehlich H, et al. (2014) Endothelial dysfunction and brachial intima-media thickness: long term cardiovascular risk with claudication related to peripheral arterial disease: a prospective analysis. PLoS One 9: e93357.

3. Jenkins NT, Padilla J, Boyle LJ, Credeur DP, Laughlin MH, et al. (2013) Disturbed blood flow acutely induces activation and apoptosis of the human vascular endothelium. Hypertension 61: 615-621.

4. Biasucci LM, Porto I, Di Vito L, De Maria GL, Leone AM, et al. (2012) Differences in microparticle release in patients with acute coronary syndrome and stable angina. Circ J 76: 2174-2182.

5. Viera AJ, Mooberry M, Key NS (2012) Microparticles in cardiovascular disease pathophysiology and outcomes. J Am Soc Hypertens 6: 243-252.

6. Leroyer AS, Anfosso F, Lacroix R, Sabatier F, Simoncini S, et al. (2010) Endothelial-derived microparticles: Biological conveyors at the crossroad of inflammation, thrombosis and angiogenesis. Thromb Haemost 104: 456-463.

7. Werner N, Wassmann S, Ahlers P, Kosiol S, Nickenig G (2006) Circulating CD31+/annexin $\mathrm{V}+$ apoptotic microparticles correlate with coronary endothelial function in patients with coronary artery disease. Arterioscler Thromb Vasc Biol 26: 112-116.

8. Tramontano AF, Lyubarova R, Tsiakos J, Palaia T, Deleon JR, et al. (2010) Circulating endothelial microparticles in diabetes mellitus. Mediators Inflamm 2010: 250476

9. Deng XL, Cao J (2012) The concentration of platelet microparticles is increased in old patients with essential hypertension complicating with coronary heart disease. Chin Med J (Engl) 125: 2602.

10. StÄpieÅ, E, Stankiewicz E, Zalewski J, Godlewski J, Zmudka K, et al. (2012) Number of microparticles generated during acute myocardial infarction and stable angina correlates with platelet activation. Arch Med Res 43: 31-35.

11. Biasucci LM, Porto I, Di Vito L, De Maria GL, Leone AM, et al. (2012) Differences in microparticle release in patients with acute coronary syndrome and stable angina. Circ J 76: 2174-2182.

12. Tan KT, Tayebjee MH, Macfadyen RJ, Lip GY, Blann AD (2005) Elevated platelet microparticles in stable coronary artery disease are unrelated to disease severity or to indices of inflammation. Platelets 16: 368-371. 